\title{
O aleitamento materno nos programas brasileiros de pós-graduação em Pediatria: perfil das dissertações e teses elaboradas de 1971 a 2006
}

\author{
Breastfeeding in Brazilian Pediatrics postgraduate programs: a profile of academic papers \\ made from 1971 to 2006
}

Lucia de Fátima G. Veloso ${ }^{1}$, João Aprígio G. Almeida²

\section{RESUMO}

Objetivo: Traçar o perfil da produção sobre o tema "aleitamento materno" na pós-graduação stricto sensu em Pediatria no Brasil.

Métodos: Análise descritiva das dissertações e teses produzidas no período de 1971 a 2006 pelos programas reconhecidos pela Coordenação de Aperfeiçoamento de Pessoal de Nível Superior.

Resultados: Os 12 programas produziram 1.494 obras, das quais $68(4,55 \%)$ utilizaram o aleitamento materno como objeto de construção, sendo 50 em nível de mestrado e 18 de doutorado. A região Sudeste detém o maior número de Programas. Houve predominância dos estudos quantitativos. A Faculdade de Medicina de Ribeirão Preto, a Universidade de São Paulo e a Universidade Federal de São Paulo foram as que mais se dedicaram ao estudo do tema. Dois programas não apresentaram estudos sobre aleitamento materno. Os programas se diferenciaram quanto à especificidade dos estudos, geralmente quantificando e descrevendo o tema sob a ótica social e biológica, mas não foram explorados aspectos culturais que envolvem a prática do aleitamento materno.

Conclusões: $\mathrm{O}$ número de obras produzidas não corresponde à importância da temática para a saúde da criança. A produção em aleitamento materno está concentrada no Sudeste, região que detém o maior número de Programas. Houve predominância dos estudos quantitativos, o que revela uma preocupação com a dimensão biológica da amamentação.

Palavras-chave: aleitamento materno; pesquisa; Pediatria.

\section{ABSTRACT}

Objective: To analyze the profile of knowledge production on breastfeeding in Brazilian pediatrics strict sensu postgraduation programs.

Methods: A descriptive analysis was carried out regarding academic studies produced between 1971 and 2006 at all programs recognized by the Coordination for Improvement of Professionals with University Level (Capes, Brasília, Brazil).

Results: The 12 programs produced 1.494 thesis, 68 of which $(4,55 \%)$ had breastfeeding as the main object: 50 master and $18 \mathrm{PhD}$ theses. The Southeast region had the largest number of programs. In terms of methodology, there was a predominance of quantitative studies. The Medical Schools of São Paulo University in Ribeirão Preto and in São Paulo together with the Federal University of São Paulo were the ones that were more dedicated to studying this issue. Two programs did not present studies on breastfeeding at all. The programs were very different regarding the specificity of their studies, generally quantifying and describing the subject under a social or a biological view, without investigating cultural aspects related to breastfeeding practice.

Conclusions: The number of academic studies produced does not match the importance of the theme for children's health. The academic production on breastfeeding is concentrated in the Southeast, the region with the largest number of programs. In terms of methodology, there was a predominance of quantitative studies, which shows a concern for the biological dimension of breastfeeding.

Key-words: breastfeeding; research; Pediatrics.
Instituição: Instituto Fernandes Figueira da Fundação Oswaldo Cruz (IFF/Fiocruz), Rio de Janeiro, RJ, Brasil

${ }^{1}$ Pediatra. Doutora em Saúde da Criança e da Mulher pelo IFF/Fiocruz, Rio de Janeiro, RJ, Brasil

2Doutor em Saúde da Criança e da Mulher pelo IFF/Fiocruz; Secretário Executivo do Programa Ibero-Americano de Bancos de Leite Humano. Chefe do Centro de Referência de Bancos de Leite Humano do IFF/Fiocruz. Professor do Programa de Pós-graduação em Saúde da Criança e da Mulher do IFF/ Fiocruz, Rio de Janeiro, RJ, Brasil
Endereço para correspondência:

Lúcia de Fátima G. Veloso

Rua Marquês de Olinda, 19, apto. 505 - Botafogo

CEP 22251-040 - Rio de Janeiro, RJ, Brasil

E-mail: luciaveloso@globo.com

Recebido em: 17/9/08

Aceito em: 17/12/08 


\section{Introdução}

Nos últimos 30 anos, a ciência tem contribuído para o resgate do aleitamento materno como prática indispensável à saúde da mulher e da criança em diferentes áreas de estudo ${ }^{(1,2)}$. Evidências das qualidades nutricionais e imunológicas do leite humano e da importância da amamentação para o fortalecimento do vínculo afetivo entre mãe e filho ${ }^{(1-4)}$ têm sido amplamente documentadas na literatura, gerando o conhecimento necessário para se estabelecerem diretrizes norteadoras dos procedimentos nesse setor ${ }^{(5-1)}$.

A literatura científica brasileira é rica em trabalhos relacionados ao aleitamento materno desenvolvidos em diferentes áreas de conhecimento, especialmente na Pediatria. Souza ${ }^{(12)}$ pesquisou os determinantes biológicos e sociais influentes na construção do conhecimento sobre a alimentação infantil na Pediatria brasileira, analisando teses e artigos publicados em revistas nacionais ${ }^{(13)}$.

Os cursos de pós-graduação iniciaram a sua trajetória na década de 1960 nas universidades brasileiras amparados pela Lei de Diretrizes e Bases da Educação de $1962^{(14-18)}$. A experiência de pós-graduação em Pediatria no Brasil tem um percurso de três décadas e o primeiro programa de pósgraduação stricto sensu foi criado em 1971 pela Faculdade de Medicina de Ribeirão Preto da Universidade de São Paulo (FMRP-USP). Até o ano de 2004, esse programa foi unidisciplinar, recebendo somente alunos com formação médica e residência médica em Pediatria. A partir de então, passou à interdisciplinaridade, recebendo profissionais não pediatras que tinham projetos de pesquisa desenvolvidos na própria instituição ${ }^{(19-21)}$.

Considerando que a construção do conhecimento científico se faz nos programas de pós-graduação stricto sensu ${ }^{(22)}$, questiona-se: qual a contribuição da pós-graduação em Pediatria brasileira para o processo de construção do conhecimento sobre aleitamento materno? Embora existam pesquisas sobre a produção científica na pós-graduação brasileira em outras áreas do conhecimento ${ }^{(15)}$, não há relato de estudos que investiguem a produção do conhecimento em aleitamento materno na pós-graduação em Pediatria. Em enfermagem, Oriá et al ${ }^{(23)}$ analisaram a produção do conhecimento sobre aleitamento materno, com ênfase nas questões teórico-metodológicos dos estudos publicados em revistas nacionais de enfermagem. Nesse sentido, o presente estudo tem como objetivo traçar o perfil da produção sobre aleitamento materno nos programas brasileiros de pós-graduação stricto sensu em Pediatria, reconhecidos pela Coordenação de
Aperfeiçoamento de Pessoal de Nível Superior (Capes), no período de 1971 a 2006.

\section{Métodos}

O estudo foi desenvolvido em três etapas: mapeamento dos programas de pós-graduação stricto sensu em Pediatria reconhecidos no Brasil; identificação e obtenção das dissertações e teses; e análise descritiva do material.

Os programas de pós-graduação stricto sensu em Pediatria e suas respectivas instituições de ensino superior foram localizados no portal da Capes, na área de Medicina, parte integrante da grande área de Ciências da Saúde.

Em seguida, solicitou-se à coordenação de cada programa uma relação completa de toda a produção. Essa relação foi a base para selecionar as dissertações e teses sobre aleitamento materno. Os estudos foram escolhidos por data de defesa, título e autor, procurando-se identificar a temática através das palavras: aleitamento materno, amamentação, leite humano, colostro, leite de transição, desmame e banco de leite humano. Por meio do Serviço de Comutação Bibliográfica entre Bibliotecas, foram obtidas cópias dos exemplares selecionados.

Cada programa de pós-graduação em Pediatria teve um período de atividade que vai do ano de criação do programa, com funcionamento unidisciplinar, até o ano em que se tornou interdisciplinar. O período de funcionamento da pós-graduação foi distinto entre os programas. Este estudo abrange o período de 1971 a 2006, datas que se referem, respectivamente, à criação do primeiro programa e ao encerramento do último programa em Pediatria.

Trata-se de um estudo transversal, no qual os dados foram tabulados e analisados segundo os preceitos da estatística descritiva e por meio de valores percentuais, buscando-se contextualizar o material a fim de compor um perfil das produções no período enfocado. O estudo foi aprovado pelo Comitê de Ética em Pesquisa da própria Instituição.

\section{Resultados}

As instituiçõos de ensino superior envolvidas neste estudo e seus respectivos programas foram encontrados em pesquisa ao site da Capes, dentro da grande área Ciências da Saúde, área Medicina, conforme a Relação de Cursos Recomendados e Reconhecidos pela Capes. No período correspondente ao estudo, somavam-se 12 programas de pós-graduação stricto sensu em Pediatria distribuídos geograficamente nas regiões 
Sul, Sudeste e Nordeste. A região Sudeste concentra o maior número de programas (oito), seguida pela região Sul, com três programas e, por último, a região Nordeste com apenas um programa. Não foram encontrados programas de pós-graduação em Pediatria (PPGP) nas regiões Norte e Centro-oeste. Durante o período estudado, três programas apresentavam somente o curso de mestrado (Tabela 1).

No período de 1971 a 2006, os programas de pós-graduação stricto sensu em Pediatria produziram 1.494 obras, sendo 1.204 dissertações de mestrado e 290 teses de doutorado. $\mathrm{O}$ aleitamento materno foi utilizado como objeto de estudo em $4,55 \%$ do total, o que corresponde a 50 dissertações e 18 teses (Tabela 2).

A análise comparativa da produção entre os programas de pós-graduação revelou que a região Sudeste concentra o maior número de dissertações e teses sobre aleitamento materno, com 54 produções $(79,4 \%)$ em seis programas (FMRP-USP, USP, UFRJ, Unifesp, UFF, UFMG), seguida pela região Sul com oito produções $(11,8 \%)$ em três programas (UFPR, UFRGS, PUC/RS) e, por último, a região Nordeste com seis produções $(8,8 \%)$ em só um programa (UFPE). Essa tendência resulta da concentração de cursos de pós-graduação stricto sensu nas respectivas regiões (Tabela 2).

A Faculdade de Medicina de Ribeirão Preto (FMRP-USP) detém o maior número de publicações sobre aleitamento materno, com $25 \%$ do total dessa temática no período estudado (Tabela 2). A análise do quantitativo da produção sobre aleitamento materno de cada programa revelou que a Faculdade de Medicina de Ribeirão Preto (FMRP-USP), a Universidade Federal de São Paulo (Unifesp) e a Universidade de São Paulo

Tabela 1 - Distribuição das instituições de ensino superior nos programas brasileiros de pós-graduação stricto sensu em Pediatria (Grande área: Ciências da Saúde; área: Medicina) reconhecidos pela Capes, com o ano de criação e o ano de extinção dos programas

\begin{tabular}{|c|c|c|c|c|c|}
\hline Programas & $\begin{array}{l}\text { Instituição de Ensino } \\
\text { Superior (IES) }\end{array}$ & $\begin{array}{l}\text { Unidade } \\
\text { Federativa }\end{array}$ & Nível & $\begin{array}{l}\text { Ano de } \\
\text { criação }\end{array}$ & $\begin{array}{l}\text { Ano de } \\
\text { extinção }\end{array}$ \\
\hline Pós-graduação em Pediatria & $\begin{array}{l}\text { Faculdade de Medicina de } \\
\text { Ribeirão Preto, Universidade } \\
\text { de São Paulo (FMRP-USP) }\end{array}$ & SP & $\mathrm{M} / \mathrm{D}$ & 1971 & 2004 \\
\hline Medicina (Pediatria) & $\begin{array}{l}\text { Universidade de São Paulo } \\
\text { (USP) }\end{array}$ & SP & M/D & 1972 & 2002 \\
\hline Medicina (Pediatria) & $\begin{array}{l}\text { Universidade Federal do Rio } \\
\text { de Janeiro (UFRJ) }\end{array}$ & RJ & $M / D$ & 1972 & 1995 \\
\hline Pós-graduação em Pediatria & $\begin{array}{l}\text { Universidade Federal de } \\
\text { Pernambuco (UFPE) }\end{array}$ & PE & M & 1973 & 2001 \\
\hline Pós-graduação em Pediatria & $\begin{array}{l}\text { Universidade Federal do } \\
\text { Paraná (UFPR) }\end{array}$ & PR & M & 1975 & 2002 \\
\hline Pós-graduação em Pediatria & $\begin{array}{l}\text { Universidade Federal de São } \\
\text { Paulo (Unifesp) }\end{array}$ & SP & $M / D$ & 1980 & 1992 \\
\hline Pós-graduação em Pediatria & $\begin{array}{l}\text { Universidade Federal } \\
\text { Fluminense (UFF) }\end{array}$ & RJ & M & 1982 & 2004 \\
\hline Pós-graduação em Pediatria & $\begin{array}{l}\text { Universidade Federal de } \\
\text { Minas Gerais (UFMG) }\end{array}$ & MG & M/D & 1987 & 2004 \\
\hline Pós-graduação em Pediatria & $\begin{array}{l}\text { Universidade Estadual de } \\
\text { Campinas (Unicamp) }\end{array}$ & SP & $M / D$ & 1988 & 2000 \\
\hline Ciências Médicas em Pediatria & $\begin{array}{l}\text { Universidade Federal do Rio } \\
\text { Grande do Sul (UFRGS) }\end{array}$ & RS & $M / D$ & 1989 & 2001 \\
\hline Medicina (Pediatria) & $\begin{array}{l}\text { Faculdade de Ciências } \\
\text { Médicas Santa Casa de São } \\
\text { Paulo (FCMSCSP) }\end{array}$ & SP & M/D & 1991 & 2006 \\
\hline Medicina (Pediatria) & $\begin{array}{l}\text { Pontifícia Universidade } \\
\text { Católica do Rio Grande do Sul } \\
\text { (PUC/RS) }\end{array}$ & RS & $M / D$ & 1995 & 2004 \\
\hline
\end{tabular}

Fonte: Capes e fontes primárias das Instituições de Ensino Superior. M: mestrado acadêmico; D: doutorado 
Tabela 2 - Distribuição da produção sobre aleitamento materno dos programas brasileiros de pós- graduação stricto sensu em Pediatria, conforme a origem institucional

\begin{tabular}{lcccc}
\hline Programas (IES) & $\begin{array}{c}\text { Total de estudos } \\
\text { (dissertações } \\
\text { e teses) }\end{array}$ & $\begin{array}{c}\text { Total da produção } \\
\text { em AM }\end{array}$ & $\begin{array}{c}\text { AM em relação ao } \\
\text { total de estudos (\%) }\end{array}$ & $\begin{array}{c}\text { AM em relação ao } \\
\text { total da produção (\%) }\end{array}$ \\
\hline FMRP-USP & 289 & 17 & 5,88 & 25,00 \\
USP & 105 & 11 & 10,48 & 16,17 \\
UFRJ & 91 & 2 & 2,2 & 2,94 \\
UFPE & 190 & 6 & 3,16 & 8,82 \\
UFPR & 78 & 1 & 1,28 & 1,47 \\
Unifesp & 178 & 16 & 8,99 & 23,53 \\
UFF & 57 & 1 & 1,75 & 1,47 \\
UFMG & 204 & 7 & 3,43 & 10,29 \\
Unicamp & 57 & 0 & 0 & 0 \\
UFRGS & 85 & 6 & 7,05 & 8,82 \\
FCMSCSP & 108 & 0 & 0 & 0 \\
PUC/RS & 52 & 1 & 1,92 & 1,47 \\
Total & $\mathbf{1 . 4 9 4}$ & $\mathbf{4 8}$ & & $\mathbf{1 0 0}$ \\
\hline
\end{tabular}

Fonte: Capes e fontes primárias das Instituições de Ensino Superior.

AM: aleitamento materno

(USP) foram as que mais se dedicaram a estudar o tema, com $25 \%, 23,5 \%, 16,2 \%$, de suas respectivas produções (Tabela 2). Os programas de pós-graduação em Pediatria da Universidade Estadual de Campinas (Unicamp) e da Faculdade de Ciências Médicas da Santa Casa de São Paulo (FCMSCSP) não dispõem de teses ou dissertações sobre aleitamento materno produzidas no período estudado (Tabela 2).

A distribuição dos estudos sobre aleitamento materno por década evidencia uma tendência crescente na produção acadêmica, com 10,3\% das obras na década de 1970,27,9\% na década de 1980 e 47,1\% das obras concentradas na década de 1990. Na Universidade Federal de Pernambuco (UFPE), o aleitamento materno se configurou como objeto de estudo para a pós-graduação desde as primeiras dissertações. A Universidade Federal do Rio de Janeiro (UFRJ) teve seu programa implantado em 1972 e somente em 1989 apresentou a primeira produção sobre aleitamento materno (Tabela 3).

Em relação ao referencial metodológico, houve uma predominância de estudos quantitativos em todos os programas de pós-graduação.

\section{Discussão}

O programa de pós-graduação em Pediatria da Unifesp, instituído em 1980 e extinto em 1992, foi responsável por 23,5\% do total de estudos sobre aleitamento materno (Tabela 2). Esse percentual expressivo é um dado importante porque mostra que não houve relação entre o número de produ- ções sobre aleitamento materno e o tempo de duração dos programas (Tabela 1), o que torna evidente que a produção dos programas depende de suas linhas de pesquisa que, por sua vez, são um reflexo do perfil institucional e do tempo necessário para a sua consolidação ${ }^{(19)}$.

Ao comparar o volume produzido nesse último período às décadas de 1970 e 1980, registrou-se um crescimento de 8,8\% na década de 1990 em relação à soma das duas anteriores $(38,2 \%)$. Essa primeira constatação, por si, já confere um importante grau de concretude à contribuição dos programas de pós-graduação stricto sensu em Pediatria na construção do conhecimento científico ${ }^{(22)}$. Em relação ao período de 2000 a 2006, fica claro que a produção limita-se aos cinco primeiros anos da década tanto para dissertações quanto para teses, o que mostra uma produção relativamente menor nesse período em consequência da reestruturação dos programas de pósgraduação em Pediatra a partir do ano 2000, com a mudança para a interdisciplinaridade e a extinção de grande parte dos programas (Tabela 1). Com a reestruturação, alguns deles passaram a se chamar de Programa de Pós-Graduação em Saúde da Criança e do Adolescente, recebendo profissionais de outras áreas do conhecimento. A tendência à interdisciplinaridade tanto docente como discente respondeu a uma exigência da Capes e foi considerada uma evolução dentro dos programas ${ }^{(19,21)}$.

Essa dinâmica observada na cronologia das publicações de dissertações e teses dos programas de Pediatria pode estar relacionada com o contexto histórico da situação do 
Tabela 3 - Distribuição quantitativa da produção acadêmica sobre aleitamento materno nos programas brasileiros de pós-graduação stricto sensu em Pediatria, por década

\begin{tabular}{lcccc}
\hline Programas & Década $\mathbf{1 9 7 0}(\mathbf{\%})$ & Década $\mathbf{1 9 8 0}(\mathbf{\%})$ & Década $\mathbf{1 9 9 0}$ (\%) & Novo milênio (\%) \\
\hline FMRP-USP & 2 & 8 & 4 & 3 \\
USP & 1 & 2 & 7 & 1 \\
UFRJ & 0 & 1 & 1 & 0 \\
UFPE & 3 & 1 & 2 & 0 \\
UFPR & 1 & 0 & 0 & 0 \\
Unifesp & 0 & 6 & 10 & 0 \\
UFF & 0 & 1 & 0 & 0 \\
UFMG & 0 & 0 & 3 & 4 \\
Unicamp & 0 & 0 & 0 & 0 \\
UFRGS & 0 & 0 & 5 & 1 \\
FCMSCSP & 0 & 0 & 0 & 0 \\
PUC/RS & 0 & 0 & 0 & 1 \\
Total & $\mathbf{7 ( 1 0 , 2 9 )}$ & $\mathbf{1 9}(\mathbf{2 7 , 9 4 )}$ & $\mathbf{3 2}(\mathbf{4 7 , 0 5 )}$ & $\mathbf{1 0}(\mathbf{1 4 , 7 1 )}$ \\
\hline
\end{tabular}

Fonte: CAPES e Fontes primárias das Instituições de Ensino Superior.

aleitamento materno no Brasil na década de 1970, com as políticas vigentes, com o desenvolvimento de uma política estatal focada na temática e, ainda, com a evolução da pósgraduação em Pediatria.

Em uma análise inicial dos trabalhos estudados realizados ao longo das décadas de 1970 (7 estudos, 10,3\%), 1980 (19 estudos, 27,9\%) e 1990 (32 estudos, 47,1\%), revelou-se uma tendência à abordagem dos seguintes temas: o aleitamento materno em determinada região; a composição bioquímica do leite humano em suas várias fases; as variáveis socioeconômicas e culturais ligadas ao aleitamento materno sobre o crescimento e o desenvolvimento de crianças a partir do nascimento; e a associação do aleitamento materno com determinadas patologias como icterícia, diarreia, desnutrição e outras.

No contexto histórico, a década de 1970 é identificada como um momento de mobilização. Em 1972 criou-se o Instituto Nacional de Alimentação e Nutrição, que tem como um de seus objetivos promover e estimular o retorno da amamentação ao seio. A opinião pública, nesse momento, começa a voltar seus interesses para os problemas causados pelo vertiginoso decréscimo da amamentação, com o desmame precoce emergindo como responsável pelas altas taxas de mortalidade infantil. A alimentação do lactente era tarefa de controle médico e predominava a distribuição de leite e o uso de fórmulas lácteas ${ }^{(1,24,25)}$.

A década de 1980 foi demarcada pela criação do Programa Nacional de Incentivo ao Aleitamento Materno, em 1981, e dos Programas de Assistência Integral a Saúde da Mulher e da criança, em 1983. Esses programas foram inovadores no sentido de destacarem ações de incentivo ao aleitamento materno. Diante das altas taxas de mortalidade infantil e des- nutrição, a Organização Mundial de Saúde (OMS) recomenda um corte na publicidade de leite em pó. Foi criado o sistema de alojamento conjunto nas unidades do SUS com a finalidade de proporcionar mais precocemente o contato entre mãe e filho, com consequente estímulo à amamentação ${ }^{(1,24,25)}$.

Entre os 19 estudos produzidos nesse período, constatouse uma abordagem positivista, com tendência à produção voltada para o contexto assistencial, o desmame e suas causas, havendo ainda um volume expressivo de estudos que tratam da composição do leite humano, com um comparativo sobre as diversas fases: colostro, leite de transição e leite maduro (11 estudos, 58,1\%). Resultados semelhantes foram encontrados nos catálogos do Centro de Estudos e Pesquisas em Enfermagem, embora as questões relacionadas ao gênero tenham começado a emergir nessa década ${ }^{(26)}$.

Na década de 1990, foram produzidos 32 estudos $(47,1 \%)$ nos programas de pós-graduação em Pediatria. Como nas duas fases anteriores, a maioria desses estudos ainda reflete um predomínio da dimensão biológica: 18 (56,3\%) abordam a composição do leite humano. Outros mantêm uma tendência voltada para o contexto assistencial, às atitudes e práticas das mães, ao desmame e suas causas, à associação do aleitamento materno com o crescimento ponderal e com patologias como icterícia e tratamento da diarreia. O conhecimento produzido nessa década, à semelhança das demais, mantém como foco a criança.

No cenário político, a década de 1990 se mostrou um momento de grandes mudanças no cenário da amamentação. Em 1991, o Fundo das Nações Unidas para a Infância e a OMS lançaram a Iniciativa Hospital Amigo da Criança. Em 
1992, o Relatório da Situação Mundial da Infância destacou e valorizou as questões de apoio legal, pessoal e emocional à mulher, posição reforçada em 1997 pelo Manual de Promoção ao Aleitamento ${ }^{(24,25-27)}$. Em 1995, criou-se no Brasil a Rede Nacional de Bancos de Leite Humano ${ }^{(28)}$, hoje conhecida como Rede Ibero-Americana de Bancos de Leite Humano, a maior do mundo. Constata-se, frente aos investimentos realizados no país, um aumento nos índices de frequência e duração da amamentação ao seio ${ }^{(27)}$.

Pode-se concluir, portanto, que o número de obras produzidas não corresponde à importância da temática para a saúde da criança. A produção em aleitamento materno está concentrada no Sudeste, região que detém o maior número de Programas. Em termos de referencial metodológico, houve predominância de estudos quantitativos, o que revela uma preocupação com a dimensão biológica da amamentação ${ }^{(13)}$.

\section{Agradecimentos}

Aos coordenadores deste trabalho e em especial às secretárias das bibliotecas e dos programas de pós-graduação em Pediatria por atenderem às solicitações do material necessário para a realização do estudo.

\section{Referências bibliográficas}

1. Alves CR, Goulart EM, Colosimo EA, Goulart LM. Risk factors for weaning among users of a primary health care unit in Belo Horizonte, Minas Gerais State, Brazil, from 1980 to 2004. Cad Saude Publica 2008;24:1355-67.

2. Cunningham AS, Jelliffe DB, Jelliffe EF. Breast-feeding and health in the 1980s: a global epidemiologic review. J Pediatr 1991;118:659-66.

3. Abrão AC. Breastfeeding: a practice that needs to be learned. Pediatria (São Paulo) 2006;28:79-80.

4. Ferreira EA, Vargas IM, Rocha SM. Um estudo bibliográfico sobre o apego mãe e filho: bases para a assistência de enfermagem pediátrica e neonatal. Rev Latino-Am Enfermagem 1998;6:111-6.

5. Braun ML, Giugliani ER, Soares ME, Giugliani C, Oliveira AP, Danelon CM. Evaluation of the impact of the Baby-Friendly Hospital Initiative on rates of breastfeeding. Am J Public Health 2003;93:1277-9.

6. Lamounier JA. Tendências do aleitamento materno no Brasil. Rev Med Minas Gerais 1999;9:59-66.

7. Organização Mundial da Saúde, UNICEF. Proteção, promoção e apoio ao aleitamento materno. Genebra: OMS; 1989.

8. WHO [homepage on the internet]. The optimal duration of exclusive breastfeeding: results of a WHO systematic review [cited 2009 Apr 22]. Available from: http://www.who.int/inf-pr-2001/en/ note2001-07.html

9. Almeida JA, Novak FR. Breastfeeding: a nature-culture hybrid. J Pediatr (Rio J) 2004;80 (Suppl 5):S119-25.

10. Brasil. Ministério da Saúde. Secretaria de Políticas Públicas de Saúde, Organização Pan Americana da Saúde. Guia alimentar para crianças menores de dois anos. Brasília: Ministério da Saúde; 2002.

11. Gota de leite [homepage on the internet]. Duas décadas de política pública no Brasil [cited 2008 Ago 20]. Available from: http://www.bvsam.icict. fiocruz. br/gotadeleite

12. Souza LM. Do leite fraco à biologia da excepcionalidade - as múltiplas faces da mesma moeda [tese de doutorado]. Rio de Janeiro (RJ): Instituto Fernandes Figueira/Fiocruz; 2003.

13. Souza LM, Almeida JA. História da alimentação do lactente no Brasil - do leite fraco à biologia da excepcionalidade. Rio de Janeiro: Revinter; 2004.

14. Trindade EM, Siqueira MD. Rumos da pesquisa: uma história da pesquisa e pósgraduação na UFPR. 20ª ed. Curitiba: Universidade Federal do Paraná; 1998.
15. Oliveira FB, Paiva MS, Kakehashi TY. A pesquisa em enfermagem no $3^{\circ}$ milênio. Acta Paul Enf 1997;10:19-25.

16. Romeo JR, Romeo Cl, Jorge VL. Estudos de pós-graduação no Brasil. [monograph on the Internet]. Rio de Janeiro: UNESCO-IESALC; 2004 [cited 2008 Apr 30]. Available from: www.ccpg.puc-rio.br/memoriapos/textosfinais/romeo2004

17. Velloso J. Mestres e doutores no país: destinos profissionais e políticas de pós-graduação. Cad Pesquisa 2004;34:583-611.

18. Dantas F. Responsabilidade social e pós-graduação no Brasil: idéias para (avali)ação. RBPG 2004;1:160-72.

19. Capes [homepage on the internet]. Coordenação de aperfeiçoamento de pessoal de nível superior [cited 2008 Apr 30]. Available from: www.capes.gov.br

20. USPRP [homepage on the internet]. Faculdade de Medicina de Ribeirão Preto [cited 2008 Apr 30]. Available from: www.fmrp.usp.br

21. Ferriani VP, Bettiol H, Barbieri MA, Mussi-Pinhata MM. From pediatrics to child and adolescent health: a brief history of the creation and review of a post-graduate program of the University of São Paulo in Ribeirão Preto. Rev Paul Pediatr 2006;24(Supl):S10-2

22. Prado ML, Gelbcke FL. Produção do conhecimento em enfermagem no Brasil: as temáticas de investigação. Rev Bras Enfermagem 2001;54:34-42.

23. Oriá MO, Glick DF, Alves MD. Trends in breastfeeding research by Brazilian nurses. Cad Saude Publica 2005;21:20-8.

24. Siqueira SR. Aleitamento materno: teses e dissertações produzidas em São Paulo e as Políticas Públicas [tese de mestrado]. São Paulo (SP): Secretaria de Estado da Saúde de São Paulo; 2005.

25. Sydrônio K. A enfermagem brasileira tecendo as redes do conhecimento no assistir em amamentação [tese de doutorado]. Rio de Janeiro (RJ): Instituto Fernandes Figueira/Fiocruz; 2006

26. Sydrônio K, Souza EO, Almeida JA. Amamentação e enfermagem: análise descritiva e relevância da produção de pós-graduação. Rev Enferm UERJ 2006;14:107-12.

27. Del Ciampo LA, Ferraz IS, Daneluzzi JC, Ricco RG, Martinelli Junior CE. Exclusive breastfeeding: of speech to practice. Pediatria (São Paulo) 2008;30:22-6

28. Brasil. Ministério da Saúde. Regulamento técnico para o funcionamento de bancos de leite humano. Resolução RDC n 171, 4 de setembro de 2006. 